\title{
Perinatal Mental Health Care across the Globe
}

\author{
Maria Muzik ${ }^{1 *}$, Vesna Pirec ${ }^{2}$, Jonathan E Handelzalts ${ }^{1,3}$, Ashlesha Bagadiaa ${ }^{4}$, Florence Gressier ${ }^{5}$, Prabha S Chandra ${ }^{6}$, Anne-Laure \\ Sutter-Dallay ${ }^{7}$ and Nine MC Glangeaud-Freudenthal ${ }^{8}$
}

${ }^{1}$ Department of Psychiatry, University of Michigan, Ann Arbor Michigan, USA

${ }^{2}$ Insight Behavioral Health Center Chicago, IL, USA

${ }^{3}$ The Academic College of Tel-Aviv Yaffo, Tel-Aviv, Israel

${ }^{4}$ Fortis La Femme Hospital, Bangalore, India

${ }^{5}$ Department of Psychiatry, Bicêtre University Hospital, Le Kremlin Bicêtre, France

${ }^{6}$ Department of Psychiatry,National Institute of Mental Health and Neurosciences, Bangalore, India

7Perinatal Psychiatry Network, Adult Psychiatry Department, Charles Perrens University Hospital and Bordeaux Population Health center, INSERM 1219, Bordeaux University, France

8INSERM, Paris, France

${ }^{*}$ Corresponding Author: Maria Muzik, Department of Psychiatry, University of Michigan, Ann Arbor Michigan, USA; Address: 4250 Plymouth Rd, Ann Arbor, 48103 Michigan, USA; Email: muzik@med.umich.edu

Received: September 20, 2019; Accepted: September 26, 2019; Published: October 03, 2019;

\begin{abstract}
The perinatal period is a vulnerable time for new mothers and their families. Broad public awareness and universal health education for all new mothers about perinatal mental illness carries significant impact. Timely screening for mental health disorders in pregnancy and postpartum is essential, followed by referrals to adequate treatments. Integrative care plays a significant role in enhancing reach and uptake. Stepped care models propose a range of treatment options based on symptoms and illness severity, and yield the most optimal outcomes for affected mothers and their children. Perinatal psychiatry, including integrative perinatal care, has over the past decades received more attention and resource allocation worldwide. But today various countries, based on their history of awareness and stigma towards perinatal illnesses, their access to overall mental health care, especially perinatal, based on insurance/payment coverage and established workforce, and based on overall national priorities and economic strength, face various challenges to establish comprehensive and systemic pathways of care for perinatal women suffering mental health challenges. Overall, there is still significant inequality across the globe both in low- and high-income countries in public awareness, infrastructure, and access, and there is still a long way to go to ease the suffering for affected perinatal women and their families.
\end{abstract}

Keywords: Perinatal Mental Health, Perinatal Care Models, Models in low- and High-Income Countries

\section{Introduction}

Perinatal mental health illness affects $10-15 \%$ of women during pregnancy and postpartum [1]. Per Diagnostic and Statistical Manual of Mental Disorders, 5th Edition, (DSM 5), the term postpartum defines women up to one month after delivery, however, in practice many postpartum mental health issues emerge during the entire first postpartum year. The last several decades have furthered our understanding regarding perinatal illness [2], primarily in two directions: (1) the recognition of the tremendous impact of untreated maternal mental illness on pregnancy and fetal outcomes, and the lasting consequences on subsequent child development [3]; and (2) the realization that there is a need for more effective models to reach impacted women on a large scale through the establishment of mental health care approaches integrated in primary care and the community [4-7]. Clearly, in order to establish optimal care for women's perinatal mental health illness, there is the need for interdisciplinary collaboration and integrative treatment approaches $[8,9]$. In the following sections, we will elaborate on these integrative perinatal treatment approaches using examples from three countries -namely India, United States, and France. The three countries located on three continents represent very different perinatal mental health systems of care. We want to shed light on the cultural and political (i.e., organization and payment scheme of health care systems) contributions to presentation, detection and treatment of perinatal mental health disorders across these three very culturally and political varied countries.

\section{Perinatal Mental Health in India}

There are currently no formal protocols in India for any form of mental health screening in the perinatal period, and the integration of mental health into maternal health is quite inadequate. Screening is limited to institutional research and non-systematic administration. This could be attributed to several reasons including, (a) poor attention to mental health by the government until recently; (b) lack of training in mental health issues among midwives and obstetricians; and (c) the limitations of existing screening tools [10]. In particular, the latter point regarding culturally valid screening tools is highly relevant. As described by Bhui \& Bhugra in 2008, women from India are more likely to have alternate explanatory models for mental illness, be 
more affected by stigma, and less likely to seek help [11]. Thus, a selfreported questionnaire is more likely to have false negatives, missing those with poor literacy and limited awareness of their own symptoms. Thus, in India, there is a need to develop simple and culturally relevant screening tools for perinatal depression and anxiety. Other culturally relevant factors are reliance on magico-religious explanatory models for mental illness, hence seeking religious healing instead of health care professionals; the cultural preference for male babies over female causing higher rates of depression with birth of a female infant; high prevalence of partner violence; mental health stigma; and low mental health literacy among professionals. Women with prior history of mental illness are stigmatized and lose autonomy in making decisions about pregnancy and childbirth. Medications are stopped prematurely due to lack of mental health awareness and poorly understood risks, without consulting mental health clinicians. Involving fathers more actively in care is needed but may sometimes be difficult, as childbirth is viewed as a woman's business and it is often the maternal family which handles childbirth. Thus, India has a long way to go to overcome some culturally based obstacles to provide evidence-based perinatal mental health care. Moreover, culture and stigma are just some of the problems Indian women face when accessing adequate care for perinatal mental health. To date, India lacks a widespread infrastructure for the provision of any mental health care. For a population of 1 billion, there are fewer than 10,000 mental health professionals, with access to specialists limited mostly to urban areas. Only about $1-2 \%$ of the total health budget is spent on mental health [12]. Additionally, the priority for maternal and infant health has still been addressing more pressing issues such as malnutrition and anemia, along with ensuring that women have access to safe hospital deliveries. Both maternal and infant mortality are very high, and continue to be priorities over mental health [13].

Over the last decade, under the National Rural Healthcare Mission the Government of India has launched several programs to improve widespread access to health care for women [14]. A major component of this program was the implementation of Community Health volunteers called Accredited Social Health Activists (ASHAs), whose main aim is to form a reliable and consistent link between the community and the health system. They are the frontline staff (usually women) to whom villagers go for education, advice and monitoring of health related issues. By reducing ignorance and superstition, ASHA workers have been responsible for timely access of emergency and institutional childbirth services, and better utilization of outpatient and diagnostic health services. The Janani Suraksha Yojana (JSY) [15] and Janani Shishu Suraksha Karyakram (JSSK) [16] were launched in 2005, to improve access to antenatal care and reduce maternal and infant mortality. Under these programs, women are encouraged to improve nutrition during the antenatal period, ensure regular antenatal care, deliver in government health care facilities, and maintain regular follow up throughout the first year of the child's life. Apart from that ASHA workers have also been trained in educating families regarding birth spacing and effective use of contraception. Each regional health center has auxiliary Nurse Midwives (ANMs), who act as supervisors and additional skilled resources for ASHA workers. Another current project is to train existing volunteer groups in identifying people with mental illness and connecting them to the right pathways of care. This program has been successfully evaluated outside of perinatal phase [17], but could in future be adopted more widely to include the perinatal population as well. Training of ASHA workers and other health professionals to screen for common mental health disorders specifically with focus on perinatal can be the next step in a wide-spread model of perinatal care

Apart from improving task workers at the ground level, National Mobile Medical Units (NMMUs) have been set up to enable access to remote areas. Mother and Child Health Wings with increased bed capacity have been sanctioned in high case load district hospitals as well as Child Health Centers which create additional beds for mothers and children. Furthermore, through nationally funded programs in many of India's states, District Hospitals have telemedicine facilities, enabling easy access to specialists in tertiary care centers, including psychiatrists. The National Informatics Centre recently launched an electronic maternal and child tracking system that registers and follows women through pregnancy, postnatal care and children from infancy to adolescents [18]; Phone based infomercials and reminders have also been used to encourage safe pregnancy and aftercare and as a result, more recently the maternal and infant mortality rates have dropped significantly [14]

All the effort noted above demonstrate India's national priority as a part of national Health Mission to establish a broad infrastructure for an impactful maternal and infant health care system, which would integrate access to perinatal mental health as well. At present perinatal mental health services are still only sparse and limited to academic institutions in a few cities, most of them restricted to outpatient services and only one inpatient mother-baby day-treatment unit (MotherBaby Hospital in Bangalore). To further support perinatal mental health, the Indian Psychiatric Society now has a section on perinatal mental health, which has been championing the cause of maternal and child mental health. A nationally convened subcommittee of psychiatrists has been assigned the task of developing guidelines for perinatal mental health, with the parallel aim to have mental health incorporated in corresponding guidelines for obstetricians and pediatricians. Liaison between obstetric and psychiatric clinicians has increased and locally developed screening systems are being trialed.

Taken all together, India has still a long road ahead to have a widespread perinatal mental health system of care and perinatal mental health integration with primary care established, but activities towards achieving the goal are underway. However, despite many obstacles based on culture (e.g., beliefs, stigma, women's rights) and other priorities (e.g., safe medical care), India is experiencing a surge of enthusiastic conversations among the responsible stakeholders about the importance of maternal and child mental health. The key is to now start putting these conversations into clinical practice and actively affect change. Successful integration and collaboration across mental health and primary care professionals and community members will reduce stigma and improve acceptance of mental health as a routine aspect of holistic healthcare in the perinatal period. This will ultimately bring positive change not only to the mother and young child, but also the whole family, and eventually the entire society. 


\section{Perinatal Mental Health in United States of America}

Mental health care throughout the United States (US) varies drastically in access and type of services available. While geographic aspects may be important (e.g., vicinity to large metropolitan centers), one major impacting factor for care delivery in the US is the presence of different tiers of payment (insurance) systems for medical care. The insurance systems range from Medicaid (covering mostly chronically disabled patients, patients with income below the poverty line, and pregnant women) to various private insurance plans, each with its own eligibility and fee structures thus creating an uneven system which leaves many persons unprotected. With the establishment of the Affordable Care Act (ACA), also known as Obama Care, in 2010, an additional 45.7 million (15.7\%) Americans became eligible for services. Under ACA all women with inadequate insurance coverage and below poverty line became eligible to receive comprehensive obstetrical care during pregnancy and up to 60 days postpartum and thus also eligible for access to quality mental health care.

From a larger politico-cultural perspective, health care inequality, especially for mental health, has unfortunately a long history in the US. There is a huge racial disparity; while most countries show a decrease in maternal mortality over past years the US is the only country with rising prevalence rate by almost 30\% (between 2000 and 2014), and this rise is entirely accounted for by African American mothers [19]. Black women tend to initiate prenatal care later in pregnancy, and have limited access to affordable care overall, as the positive outcomes of ACA are still lagging. Moreover, the US is a racial and cultural "melting pot" in which attitudes towards mental health in general (and in particular towards perinatal mental health) varies widely based on cultural and economic background. Externalized stigma is particularly predominant in some subcultures preventing women from identifying and seeking help. This is true for African American, Latina, Asian or Arabic women, as well as the many other immigrant women from all over the world. Often women feel the need to identify as "strong" because they fear disclosure of any emotional problems may lead to losing child custody. Additionally, immigrants from many parts of the world have often limited knowledge of mental health, and in particular feel ashamed to disclose emotional problems in motherhood. Cultural sensitivity, access to interpreters with awareness of culture-bound obstacles, and provision of new engagement and treatment models is critical. One of such initiatives to overcome cultural barriers and the access gap was the establishment of the Centering Pregnancy group model as a nationwide initiative allowing for groups of women to experience pregnancy and postpartum health care visits together as a group thus promoting social support; however, results from this initiative are not yet known [20].

Despite of all these aforementioned obstacles, maternal perinatal mental health continues to be an important public health concern, and clinicians, professionals, and families alike are pushing for improvements. In an attempt to identify more women suffering from perinatal illness, primary care settings (i.e., family medicine, obstetrics and pediatrics) were recognized as an ideal place in which screening can be conducted. Already in 2009 the US Preventive Task Force (USPSTF) recommended screening adults for depression in general [21]. As a response to this initiative, several US states launched legislations mandating postpartum depression screening [22]. Additionally, The Mother's Act, a federal legislation passed in 2010, offered government support for research that would support perinatal screening. Since many women in the US receive some level of health care during pregnancy, obstetricians and family practitioners, who deliver such care during pregnancy, were those identified as most feasible to conduct screening [23]. To support this initiative the American Congress of Obstetricians and Gynecologists (ACOG) came out to support such screening for perinatal mental illness in pregnancy and postpartum (ACOG,) [24]. Similarly, the American Academy of Pediatrics (AAP) also made a recommendation that all pediatricians should screen mothers when they present with their child to the 6-months well-baby visit.

However, soon it became apparent that screening without appropriate opportunity for treatment is not effective [25]. Nevertheless, despite recommendations put in place through national professional associations, many women were still not screened for peripartum depression or anxiety. Factors most commonly identified to interfere with follow through with the recommendations were as follows: (1) limited time and lack of reimbursement from insurances for screening; (2) illiteracy regarding perinatal mental illness and how to approach women with perinatal mental illness; (3) insufficient referral resources providing adequate quality perinatal mental health care.

This led to the proposition of several published models. First, models where detection and treatment can take place directly through obstetricians caring for pregnant women (the Perinatal Depression Management Program (PDMR) or Step Care Model [26]. in this model initial universal screening is performed with validated tools for perinatal depression during antenatal care visits. The providers would be educated about perinatal mental illness and be able to approach identified high-risk patients (those who scored above cut off) to complete a brief ( 5-6 minutes) psychiatric assessment. These providers would also receive education about prescribing medicines for depression and anxiety (e.g., SSRIs), which have a low side effect profile and are safe during pregnancy and breastfeeding, so can easily be prescribed by non-psychiatrists. Patients with mild and moderate depression and anxiety disorder would be treated in the primary care setting. In contrast, patients with severe symptoms of mental illness would be quickly identified and referred out to mental health providers for specialty care. Despite this approach being well described and showing benefits [26], the uptake across the US has not been uniform.

Secondly, detection and referrals can take place in pediatric offices [27]. In this setting, new mothers can be successfully identified using standard screening tools such the Edinburgh Post depression scale (EPDS) [28], and if identified at risk could receive on-site counseling or, if medications are needed, referred out to specialty care. Pediatric primary care providers, unlike adult healthcare providers, have the most frequent contact with postpartum women through well-child visits. Well-child visits thus present an unparalleled opportunity to detect and intervene with postpartum mental illness. 
Both these above mentioned models are most effective when mental health providers are embedded into the primary care settings allowing immediate and direct access to counseling [29,30]. Thus, obstetric and pediatric offices may have a mental health worker (often clinicians with social work background) available on site who can further assess all women who screened positive. Women at risk are then immediately identified and provided with either brief counseling by the social worker, or sent to see a psychiatrist either in the same clinic (available during special hours) or referred outside to a psychiatric clinic. However, again, lack of a uniformed health care system and one common health has made budget this great idea challenging to implement. Additionally, there are still large gaps in knowledge among women's reproductive health care providers on how to optimally and safely screen and treat perinatal women with mental health disorders, and more work has to be done.

Depending on the severity of presenting symptoms among women with perinatal mental health problems, several different levels of care are available: outpatient, day hospital (or else called Partial Hospital) or inpatient treatments. Contrary to inpatient mother baby units (MBU) being widely spread through out the world (e.g., Europe, Australia) over the last 50 years, the US health system does not support resources necessary to keep a baby overnight on an adult psychiatry unit. Currently there exists only one small Perinatal Psychiatry Inpatient unit established in 2011 at the University of North Carolina that provides specialty perinatal inpatient care; Infants are allowed to stay on the unit during the day only. Another level of care is motherbaby partial hospital, where affected women (pregnant or postpartum) attend the program (if possible accompanied by their baby) during the day time for approximately 6 hours, and spend the night back at home or in an unsupervised sleeping arrangement (e.g., hotel). The first such unit opened in 2000 at Brown University [31], and since many more such units opened across the US. Many Academic settings have established specialized outpatient perinatal clinics and offer multimodal treatments. These outpatient clinics may offer also Intensive Outpatient Programs (IOPs), which are more intensive daytime groups of usually 3 hours.

Finally, several programs have been developed to provide therapeutic support for perinatal women with young children and their families who are poor or at high risk based on certain characteristics (e.g., poor, single mothers, young age etc). These programs are mostly government-funded and paid for by Medicaid. Examples of such interventions providing therapeutic support around mental health, coping with stressors and if applicable with parenting are the NurseFamily Partnership (NFP) [32], or Healthy Families America (HFA) [33] just to name a few. Each State in the US may grant access to a variety of these programs. Specific trauma-focused psychotherapy group interventions for perinatal mothers with interpersonal trauma histories were recently also developed, including TARGET, a program for mothers with depression, PTSD and substance use disorders (SUDs) [34]; Mom Power, a 13 session program for mothers with interpersonal trauma history, mental health and parenting problems [35]; or Survivor Moms' Companion (SMC), a psychoeducational program for pregnant trauma-survivor women with PTSD and depression delivered in pregnancy [36]. In summary, while perinatal mental health initiatives have started to be more widely implemented across the US, there are still gaps. While over the last years screening for perinatal depression has been mandatory in many States, it has not adequately improved referral and treatment outcomes. Furthermore, gains in perinatal mental health across the US are inconsistent, and large groups, especially Minority women and women living in poverty, have limited access to quality care despite several government-funded programs. Integrative perinatal care model while known to be effective, are still unevenly implemented across the US.

\section{Perinatal Mental Health in Europe: exemplified by France}

France has a long tradition of perinatal mental health dating back to the $19^{\text {th }}$ century. Esquirol described women admitted after childbirth to the asylums, and shortly after, in 1858, Louis-Victor Marcé published the first monograph of perinatal psychiatric pathology. In more recent history, after World War II in 1945, France developed a nation-wide, free of charge, community-based Mother and Child Protection Service (PMI) aimed to support families during pregnancy until the child's $3^{\text {rd }}$ birthday. This service provides home visits by PMI midwives and nursery nurses if necessary, and preventive follow-up visits for children during which developmental screening and are conducted vaccinations, and in this context maternal mental health can also be screened for and referrals to specialty care placed. In addition, there are large governmental programs and recommendations in place supporting perinatal mental health. Recommendations have been established to conduct early prenatal interviews for all pregnant women and future fathers listening to their concerns, needs, difficulties and expectations for pregnancy, childbirth and future parenting, with the goal of defining the best medicopsycho-social follow-up and support for the pregnancy. However, a national survey in 2010 showed that only $40 \%$ of the pregnant women receive this early comprehensive prenatal interview, and that, it is mainly mothers with low psychosocial risk that attend this interview and get benefit from it [37]. However, some kind of psychosocial screening of high-risk women during pregnancy and/or in first 3 days postpartum is performed in all regions across France. Additionally, liaison psychiatry networks connected to obstetrical Maternity Units have been established providing psychiatric support to primary care treating pregnant and postpartum women on a wide scale.

Perinatal mental health services range in France, similarly to the US, from inpatient care, partial hospital day-treatment units and outpatient psychiatric clinics, to integrated care in primary care settings. In France, pathway to care has no gatekeeper (and women in France may directly access specialized perinatal psychiatry). In contrast to the US, the number of facilities per capita is much higher in France, and there are many specialized inpatient psychiatric units for joint mother-baby admissions and psychiatric care, run by a multidisciplinary staff. In this regard, France resembles more the broad range of perinatal psychiatry treatment options similar to the UK, Australia and a few others, mostly European, countries. In case of maternal severe psychiatric illness after the childbirth, the mother and her infant can be jointly admitted to an inpatient mother baby unit $(\mathrm{MBU})$, where the mother receives psychiatric attention, while 
simultaneously the child's safety, developmental needs and the motherinfant bond are supported [38]. Women (and infants) hospitalized in MBUs have a mean length of stay of about 9 weeks, and improvement rates are very high (69\%-82\% at discharge depending on primary diagnosis [39]. Discharges from MBUs and subsequent follow up are collaboratively prepared with the women, her social support system, and her treatment providers [40]. In addition, the outpatient perinatal system of care in France, in outpatient academic or governmental clinics or through private practice perinatal psychiatrists, is wide spread and comprehensively covering the country.

In summary, perinatal psychiatry in France (and Europe overall) has a long tradition, and the perinatal psychiatry infrastructure is well established. The offerings span a broad range of stepped care models. Guided by a multilevel administrative health care coordination system, France's perinatal care spans from mother (-father)-infant psychotherapies offered in outpatient settings to joint mother-baby psychiatric hospitalizations in specialty MBUs. Similarly, to US and India, recent efforts are to enhance preventive public health care models, through integration of perinatal psychiatric care into primary care settings.

\section{Conclusion}

Perinatal psychiatry, including integrative perinatal care, has over the past decades received more attention across the globe, both in high- and middle/low- income countries, such as US, France or India. More attention and resources specifically to perinatal mental health are allocated worldwide, yet still the various countries based on historical awareness and stigma towards perinatal illnesses, access to overall mental health care, types of insurance/payment coverage, access to an established and educated workforce, and based on overall national priorities and economic strength, face different challenges in establishment of comprehensive and systemic pathways of care for perinatal women suffering mental health challenges. Overall, there is still significant inequality across the globe in public awareness, infrastructure, and access. In order to ease the suffering of affected women and their families during this critical time period of a new beginning, such that pregnancy and postpartum, we are tasked to do better, and establish more comprehensive and accessible perinatal mental health systems of care.

\section{References}

1. Fisher J, Meena Cabral de Mello, b Vikram Patel, c Atif Rahman, d Thach Tran, et al. (2012) Prevalence and determinants of common perinatal mental disorders in women in low- and lower-middle-income countries: a systematic review. Bulletin of the World Health Organisation 90: 139-149.

2. Muzik M, Thelen K, Rosenblum K (2011) Perinatal Depression: Detection and Treatment. A review. Neuropsychiatry 1: 179-195.

3. Waxler E, Thelen K, Muzik M (2011) Maternal Perinatal Depression - Impact on Infant and Child Development. European Psychiatric Review 4: 41-44.

4. Misra DP, Guyer B, Allston A (2002) Integrated perinatal health framework. A multiple determinants model with a life span approach. American Journal of Preventive Medecine 25: 65-75.

5. Yonkers KA, Smith MV, Lin H, Howell HB, Shao L, et al. (2009) Depression screening of perinatal women: An evaluation of the Healthy Start depression initiative. Psychiatric Services 60: 322-328.

6. Yonkers KA, Wisner KL, Stewart DE, Oberlander TF, Dell DL, et al. (2009) The management of depression during pregnancy: a report from the American Psychiatric Association and the American College of Obstetricians and Gynecologists. General Hospital Psychiatry 31: 403-413.
7. Thomas M, Hutchison M, Castro G, Nau M, Shumway M, et al. (2017) Meeting Women Where They Are: Integration of Care As the Foundation of Treatment for At-Risk Pregnant and Postpartum Women. Matern Child Health J 21: 452-457. [crossref]

8. Myors KA, Michelle Cleary, Maree Johnson, Virginia Schmied (2013) Collaboration and integrated services for perinatal mental health: an integrative review. Child and Adolescent Mental Health 18: 1-10.

9. Milgrom J1, Gemmill AW2 (2014) Screening for perinatal depression. Best Pract Res Clin Obstet Gynaecol 28: 13-23. [crossref]

10. Hema D, Girish N, Ramapriya, Chandra Prabha, Gururaj G (2008) Use of Edinburgh Postnatal Depression Scale (EPDS) in a private obstetrics setting. Journal of Obstetrics and Gynecology of India 58: 41-44.

11. Bhui K, Bhugra D (2008) Explanatory models for mental distress: implications for clinical practice and research. British Journal of Psychiatry 181: 6-7.

12. Patel V, Xiao S, Chen H, Hanna F, Jotheeswaran AT, et al. (2016) The magnitude of and health system responses to the mental health treatment gap in adults in India and China. Lancet 388: 3074-3084. [crossref]

13. Doke PP1 (2016) Decline and disparity in maternal mortality in pre- and postnational health mission period in India. Indian J Public Health 60: 294-297. [crossref]

14. NagarajanS, Vinod K. Paul, Namrata Yadav, Shuchita Gupta (2015) The National Rural Health Mission in India: its impact on maternal, neonatal, and infant mortality. Seminars in Fetal \& Neonatal Medicine 20: 315-320.

15. Gupta SK, Dinesh K. Pal, Rajesh Tiwari, Rajesh Garg, Ashish K. Shrivastava, et al. (2012) Impact of Janani Suraksha Yojana on institutional delivery rate and maternal morbidity and mortality: an observational study in India. Journal of Health, Population and Nutrition 30: 464-471.

16. Salve HR, Charlette L, Kankaria A, Rai SK, Krishnan A, et al. (2017) Improving Access to Institutional Delivery through Janani Shishu Suraksha Karyakram: Evidence from Rural Haryana, North India. Indian J Community Med 42: 73-76. [crossref]

17. Patel V, Weiss HA, Chowdhary N, Naik S, Pednekar S, et al. (2010) Effectiveness of an intervention led by lay health counsellors for depressive and anxiety disorders in primary care in Goa, India (MANAS): a cluster randomised controlled trial. Lancet 376: 2086-2095.

18. Grollman C, Arregoces L, Martínez-Álvarez M, Pitt C, Mills A, et al. (2017) 11 years of tracking aid to reproductive, maternal, newborn, and child health: estimates and analysis for 2003-13 from the Countdown to 2015. Lancet Glob Health 5: e104-104e114. [crossref]

19. MoaddabA, Dildy GA, Brown HL, Bateni ZH, Belfort MA, Sangi-Haghpeykar H, et al. (2016) Health care disparity and state-specific pregnancy-related mortality in the United States, 2005-2014. Obstetrics and Gynecology 128: 869-875

20. Catling CJ, Medley N, Foureur M, Ryan C, Leap N, et al. (2015) Group versus conventional antenatal care for women. Cochrane Database Syst Rev 2: CD007622. [crossref]

21. U.S. Preventive Services Task Force (2009) Preventive Services Task Force. Screening for depression in adults: U.S. preventive services task force recommendation statement. Annals of Internal Medicine 151: 784-792.

22. Perinatal Depression Information Network: Legislation, 2011 Available at Pdinfonetwork.org Accessed January 23, 2012.

23. Lee LC, Casanueva CE, Martin SL (2005) Depression among female family planning patients: Prevalence, risk factors, and use of mental health services. Journal of Women's Health 14: 225-232.

24. American College of Obstetrics and Gynecologists (AOCG) (2010) Women's Health Care Physicians. Screening for depression during and after pregnancy. Committee opinion Pg No: 453.

25. Thombs BD, Arthurs E, Coronado-Montoya S, Roseman M, Delisle VC, et al (2014) Depression screening and patient outcomes in pregnancy or postpartum: A systematic review. Journal of Psychosomatic Research 76: 433-446.

26. Miller LJ, Shade M, Vasireddy V (2009) Beyond screening: Assessment of perinatal depression in a perinatal care setting. Archives of women's Mental Health 12: 329334.

27. Olin Su-chin S, et al. Journal of Women's Health, 2017, Jul 4 Epub ahead of print. (https://doi-org.proxy.lib.umich.edu/10.1089/jwh.2016.6089)

28. Cox JL, Holden JM, Sagovsky R (1987) Detection of postnatal depression. Development of the 10-item Edinburgh Postnatal Depression Scale. British Journal of Psychiatry 150: 782-786.

29. Schmied V, Kim Psaila, Cathrine Fowler, Sue Kruske, et al. (2010) The nature and impact of collaboration and integrated service delivery for pregnant women, children and families. Journal of Clinical Nursing 19: 3516-3526.

30. Hayes BA (2010) From 'postnatal depression' to 'perinatal anxiety and depression': key points of the National Perinatal Depression Plan for nurses and midwives in Australian primary health care settings. Contemp Nurse 35: 58-67. [crossref] 
31. Howard M, Battle CL, Pearlstein T, Rosene-Montella K (2006) A psychiatric mother-baby day hospital for pregnant and postpartum women. Archives of women's mental health 9: 213-218.

32. Olds DL (2010) The nurse-family partnership: From trials to practice. In A. Reynolds A, et al (eds.), Childhood programs and practices in the first decade of life: A human capital integration.). New York, NY: Cambridge University Press Pg No: 49-75.

33. Holton JK, Harding K (2007) Healthy Families America ${ }^{\circledR}$ : Ruminations on Implementing a Home Visitation Program to Prevent Child Maltreatment. Journal of prevention \& intervention in the community 34: 13-38.

34. Ford JD, Russo E (2006) Trauma-focused, present-centered, emotional selfregulation approach to integrated treatment for posttraumatic stress and addiction: trauma adaptive recovery group education and therapy (TARGET). American Journal of Psychotherapy 60: 335-355.

35. Rosenblum KL, Maria Muzik, Diana Morelen, Emily Alfafara, Nicole Miller, et al. (2017) A community-based randomized controlled trial of Mom Power parenting intervention for mothers with interpersonal trauma histories and their young children. Archives of Women's Mental Health Jun 25 on line. (DOI: 10.1007/ s00737-017-0734-9).
36. Seng JS (2011) Integrating care for posttraumatic stress and physical comorbidities: the road is clear. Journal of American Psychiatry Nurses Association 17: 376-377.

37. Bales M, Pambrun E, Melchior M, Glangeaud-Freudenthal NM, Charles MA, et al. (2015) Prenatal psychological distress and access to mental health care in the ELFE cohort. European psychiatry 30: 322-328.

38. Brockington I, Butterworth R, Glangeaud-Freudenthal NM-C (2017) An international position paper on mother-infant (perinatal) mental health, with guidelines for clinical practice. Archives of Women's Mental Health 20: 113-120.

39. Glangeaud-Freudenthal NM-C, Sutter AL, Thieulin AC, Dagens-Lafont V, Zimmermann MA, et al. (2011) Inpatient mother and child postpartum psychiatric care: factors associated with improvement in maternal mental health. European Psychiatry 26: 215-223.

40. Glangeaud-Freudenthal N.M.-C, Sutter-Dallay AL, Thieulin AC, Dagens V, Zimmermann MA, et al.(2013) Predictors of infant foster care in cases of maternal psychiatric disorders. Social Psychiatry and Psychiatric Epidemiology 48: 553561 .

\section{Citation:}

Muzik M, Pirec V, Handelzalts JE, Bagadia A, Gressier F, Chandra PS, Sutter-Dallay A, Glangeaud-Freudenthal NMC (2019) Perinatal Mental Health Care across the Globe. ARCH Women Health Care Volume 2(5): 1-6. 